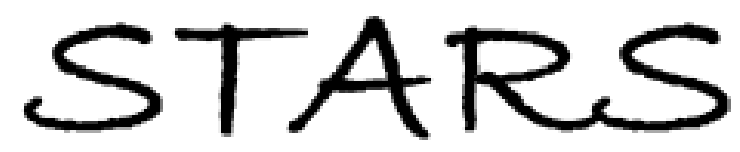

University of Central Florida

STARS

$1-1-2008$

\title{
Thin cell fringe-field-switching liquid crystal display with a chiral dopant
}

Zhibing Ge

University of Central Florida

Shin-Tson wU

University of Central Florida

Seong Su Kim

Ji Woong Park

Seung Hee Lee

Find similar works at: https://stars.library.ucf.edu/facultybib2000

University of Central Florida Libraries http://library.ucf.edu

This Article is brought to you for free and open access by the Faculty Bibliography at STARS. It has been accepted for inclusion in Faculty Bibliography 2000 s by an authorized administrator of STARS. For more information, please contact STARS@ucf.edu.

\section{Recommended Citation}

Ge, Zhibing; wU, Shin-Tson; Kim, Seong Su; Park, Ji Woong; and Lee, Seung Hee, "Thin cell fringe-fieldswitching liquid crystal display with a chiral dopant" (2008). Faculty Bibliography 2000s. 361. https://stars.library.ucf.edu/facultybib2000/361






\section{Thin cell fringe-field-switching liquid crystal display with a chiral dopant}

Cite as: Appl. Phys. Lett. 92, 181109 (2008); https://doi.org/10.1063/1.2918838

Submitted: 09 March 2008 . Accepted: 10 April 2008 . Published Online: 08 May 2008

Zhibing Ge, Shin-Tson Wu, Seong Su Kim, Ji Woong Park, and Seung Hee Lee

\section{ARTICLES YOU MAY BE INTERESTED IN}

Electro-optic characteristics and switching principle of a nematic liquid crystal cell controlled by fringe-field switching

Applied Physics Letters 73, 2881 (1998); https://doi.org/10.1063/1.122617

Electro-optical characteristics and switching behavior of the in-plane switching mode Applied Physics Letters 67, 3895 (1995); https://doi.org/10.1063/1.115309

Electro-optics of polymer-stabilized blue phase liquid crystal displays Applied Physics Letters 94, 101104 (2009); https://doi.org/10.1063/1.3097355

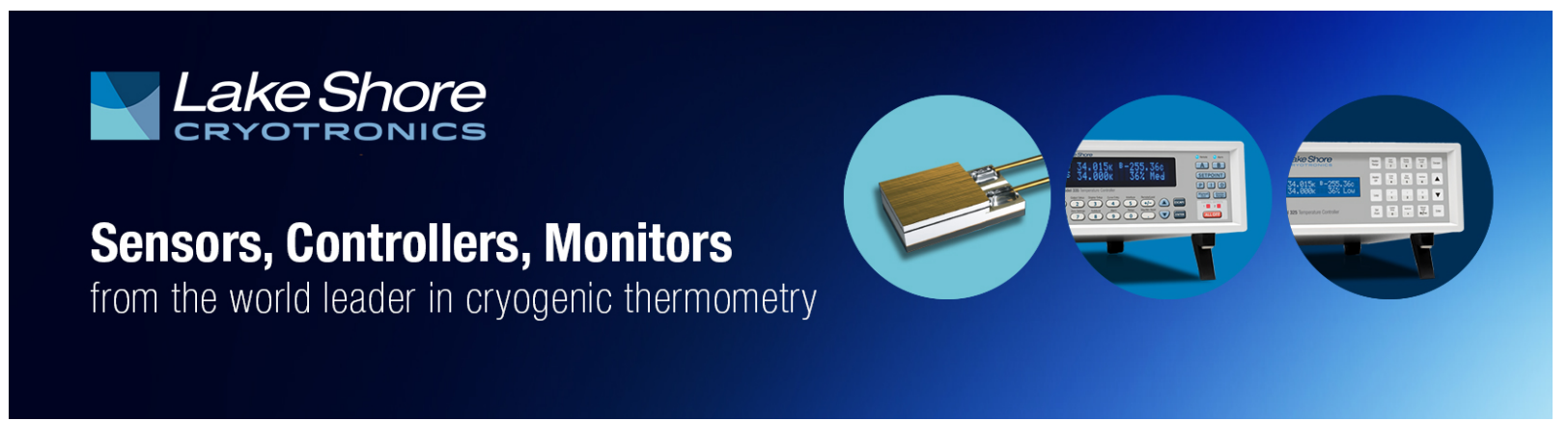




\title{
Thin cell fringe-field-switching liquid crystal display with a chiral dopant
}

\author{
Zhibing Ge, ${ }^{1}$ Shin-Tson Wu, ${ }^{1, a)}$ Seong Su Kim, ${ }^{2}$ Ji Woong Park, ${ }^{2}$ and Seung Hee Lee ${ }^{2}$ \\ ${ }_{1}^{1}$ College of Optics and Photonics, University of Central Florida, Orlando, Florida 32816, USA \\ ${ }^{2}$ Department of Polymer Nano-Science and Engineering, Chonbuk National University, Chonju, \\ Chonbuk 561-756, Republic of Korea
}

(Received 9 March 2008; accepted 10 April 2008; published online 8 May 2008)

\begin{abstract}
A fringe-field-switching (FFS) liquid crystal display (LCD) using a thin cell doped with a reverse-handed chiral compound is proposed. Such a FFS LCD exhibits a fast response time $(\sim 8 \mathrm{~ms})$, high transmittance $(>90 \%)$, low operating voltage $\left(5 \mathrm{~V}_{\mathrm{rms}}\right)$, and intrinsically wide viewing angle. Its application for LCD televisions in order to reduce image blurring is emphasized. () 2008 American Institute of Physics. [DOI: 10.1063/1.2918838]
\end{abstract}

Fast response time is critically important for reducing the motion blurs of liquid crystal displays (LCDs), especially for televisions (TVs) and portable video players. The LC response time is governed by the cell gap $(d)$, viscoelastic coefficient, anchoring energy, and applied voltage. ${ }^{1}$ Besides the efforts on synthesizing low viscosity LC materials, reducing the cell gap remains to be the most straightforward approach. $^{2-4}$ For direct-view LCD TVs, a typical cell gap is about $4 \mu \mathrm{m}$ and the trend is toward thinner cell gaps. In addition to fast response time, wide viewing angle, high transmittance, and low driving voltage are also crucial. Presently, optical film-compensated multidomain vertical alignment $^{5}$ and homogenous alignment in-plane switching ${ }^{6,7}$ or fringe-field-switching (FFS) (Ref. 8) LCDs are the two major choices for wide-view technologies. For FFS mode, its transmittance has approached or even exceeded that of a twisted nematic (TN) (Ref. 9) LCD by reducing the electrode width and gap. However, to obtain a high transmittance in a thin FFS cell $(\sim 2 \mu \mathrm{m})$, both high LC birefringence and fine electrode resolution $(\sim 1 \mu \mathrm{m})$ are required. Despite the hardship of manufacturing, such a fine electrode width and gap leads to a significantly increased driving voltage. ${ }^{10}$ Thus, there is an urgent need to develop better approaches for not only achieving fast response time but also keeping high transmittance, low operating voltage, and reasonable photolithographic resolution.

In this letter, we first analyze the LC director distribution of a FFS LC cell at various positions near the electrodes and slits and their effects to the polarization change through Poincaré sphere. We then propose a thin $(d=2 \mu \mathrm{m})$ FFS cell with a reverse-handed chiral dopant to obtain the desired LC reorientation profile. The improved FFS cell exhibits a fast response time, high transmittance $(>90 \%$, referenced to the value of two parallel polarizers), and low operating voltage $\left(\sim 5 \mathrm{~V}_{\mathrm{rms}}\right)$ by using a $2 \mu \mathrm{m}$ electrode width and $3 \mu \mathrm{m}$ electrode gap.

Figure 1 shows the FFS device structure using a positive dielectric anisotropic $(\Delta \varepsilon>0)$ LC material, calculated equal potential lines (dashed lines), and corresponding transmittance (top) in a voltage-on state. The LC directors are initially homogeneously aligned between two substrates. On the bottom substrate, a transparent plane electrode is formed as the common electrode and stripe-shaped pixel electrodes are formed above the common electrode. The electrode width is

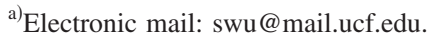

$w$ and gap is $g$. The initial LC rubbing direction is set parallel to the transmission axis of the bottom polarizer (not shown here), and the top linear polarizer (not shown here) is crossed to the bottom one. Therefore, when there is no voltage applied, the incident light from the bottom polarizer keeps its linear polarization when transversing the LC cell, and is absorbed by the top crossed polarizer resulting in a good dark state. On the other hand, when a high voltage is applied between the pixel and common electrodes, strong fringe fields with both horizontal and vertical components are generated. As the equal potential lines show in Fig. 1, the fringe fields near the pixel electrode edges have the strongest horizontal fields, and those near the centers of electrodes and slits have almost merely vertical fields. Under such an electric field profile, the LC directors near the electrode edges twist the most, and the LC directors at other regions experience a less twist and a small tilt. As a result, the transmittance near the electrode edges is higher than those in the symmetric centers above the electrodes and slits.

It is known that decreasing the electrode width and gap will increase the transmittance. However, applying fine patterns in a thin FFS cell has big tradeoffs. Table I compares the maximum transmittance, operation voltage, rise time, and decay time of several FFS cells with different cell gaps and

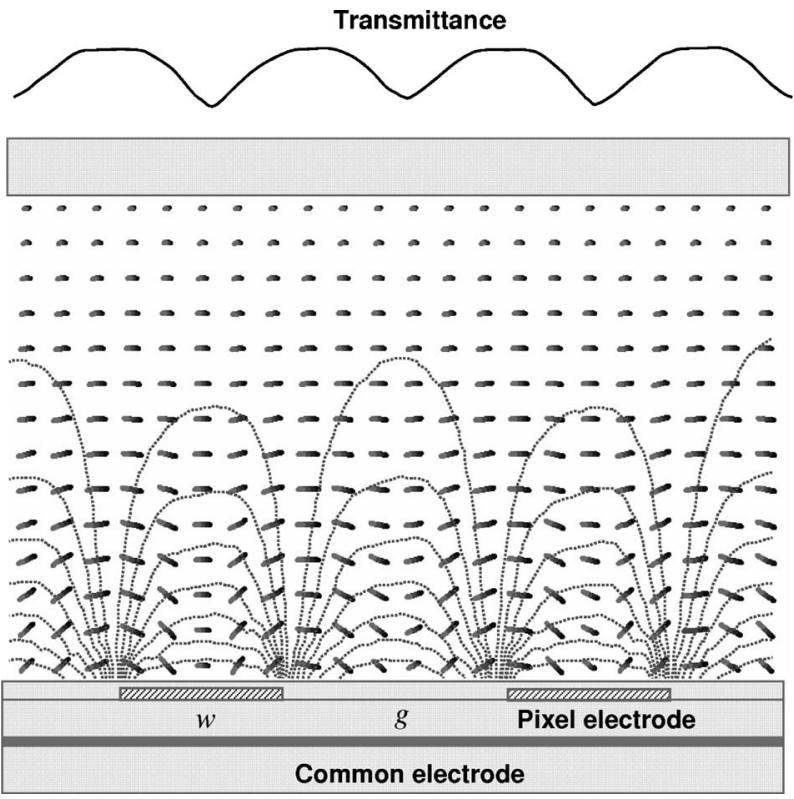

FIG. 1. Schematic structure of the FFS LCD, equal potential distribution (dashed lines), and corresponding transmittance (top) in a voltage-on state. 
TABLE I. Simulated electro-optic properties of different FFS cells without any chiral dopant.

\begin{tabular}{lcccccc}
\hline \hline & \multirow{2}{*}{$\begin{array}{c}w=3 \mu \mathrm{m}, g=4.5 \mu \mathrm{m} \\
d=2 \mu \mathrm{m}\end{array}$} & $d=4 \mu \mathrm{m}$ & \multicolumn{2}{c}{$w=2 \mu \mathrm{m}, g=3 \mu \mathrm{m}$} & \multicolumn{2}{c}{$w=1 \mu \mathrm{m}, g=1.5 \mu \mathrm{m}$} \\
& & $d=2 \mu \mathrm{m}$ & $d=4 \mu \mathrm{m}$ & $d=2 \mu \mathrm{m}$ & $d=4 \mu \mathrm{m}$ \\
\hline$T_{\max }(\%)$ & 77.5 & 90.3 & 86.2 & 95.2 & 95.6 & 98.8 \\
$V_{\text {op }}\left(V_{\text {rms }}\right)$ & 4.0 & 4.5 & 4.5 & 5.0 & 7.0 & 8.0 \\
$t_{\text {rise }}(\mathrm{ms})$ & 7.6 & 25.7 & 7.0 & 28.0 & 6.8 & 30.2 \\
$t_{\text {decay }}(\mathrm{ms})$ & 8.3 & 32.7 & 8.5 & 33.2 & 8.6 & 34.3 \\
\hline \hline
\end{tabular}

electrode dimensions. In Table I, the parameters of the LC material used for the $4 \mu \mathrm{m}$ cells are listed as follows: elastic constants $K_{11}=9.7 \mathrm{pN}, K_{22}=5.2 \mathrm{pN}$, and $K_{33}=13.3 \mathrm{pN}$, dielectric anisotropy $\Delta \varepsilon=+8.2$, birefringence $\Delta n=0.1$ at $\lambda$ $=550 \mathrm{~nm}$, and rotational viscosity $\gamma_{1}=84 \mathrm{mPa}$. Here, for the $2 \mu \mathrm{m}$ cells, the birefringence is assumed to be 0.2 while other LC parameters remain unchanged. The surface rubbing angle of the LC layer is set at $80^{\circ}$ with a pretilt angle of $2^{\circ}$, and strong anchoring is assumed on both surfaces. Besides, the insulator layer between the pixel electrode and common electrode is $\mathrm{SiO}_{x}$ with a dielectric constant of 4.5 and a thickness of $250 \mathrm{~nm}$. The LC director distribution is calculated by a commercial software 2DIMMOS (from autronicMELCHERS, Germany) and the optical properties are calculated using the $2 \times 2$ extended Jones matrix method. ${ }^{11,12}$ From Table I, with the typical electrode width at $3 \mu \mathrm{m}$ and gap at $4.5 \mu \mathrm{m}$, the transmittance is $\sim 90.3 \%$ at an operation voltage $V_{\mathrm{op}} \sim 4.5 \mathrm{~V}_{\text {rms }}$ for a $4 \mu \mathrm{m} \mathrm{LC}$ cell. By decreasing the electrode dimensions to $w=1 \mu \mathrm{m}$ and $g=1.5 \mu \mathrm{m}$, the transmittance is enhanced to $\sim 98.8 \%$, however, the driving voltage is increased from 4.5 to $\sim 8 \mathrm{~V}_{\text {rms }}$. More importantly, for these $4 \mu \mathrm{m}$ cells, their rise time is $\sim 28 \mathrm{~ms}$ and decay time is $\sim 33 \mathrm{~ms}$, which fall short for video applications, even with the overdrive and undershoot method. Here, the response time is defined as the transmittance changes from $10 \%$ to $90 \%$. By reducing the cell gap to $2 \mu \mathrm{m}$, the rise time is reduced to $\sim 7 \mathrm{~ms}$ and decay time to $\sim 8 \mathrm{~ms}$, which is $\sim 4 \times$ faster than the $4 \mu \mathrm{m}$ cell.

In addition, from Table I to obtain a good balance between high transmittance and low driving voltage, the electrode width $w \sim 2 \mu \mathrm{m}$ and gap $g \sim 3 \mu \mathrm{m}$ is preferred based on the present photolithographic precision. However, for a $2 \mu \mathrm{m}$ thin cell gap, the maximum transmittance is only $86.2 \%$. In order to find the origins of this low transmittance, the LC director distributions at different cell positions (from

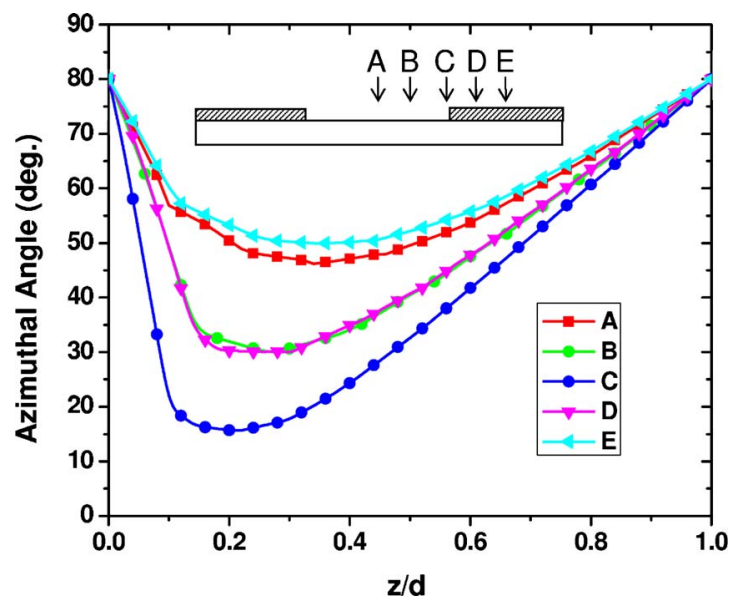

FIG. 2. (Color online) Azimuthal angle distributions at different cell positions without a chiral dopant.
A to E) are plotted in Fig. 2. From Fig. 2, the LC twist is mainly confined near the bottom of the cell $(0.2<z / d$ $<0.4)$ and is gradually restored back to the initial rubbing direction by the top surface anchoring force. The cell appears like two TN cells in series but with reversed twist sense. From Fig. 1, horizontal fields are the strongest near the electrode edges, resulting in a maximum azimuthal angle change of $\sim 65^{\circ}$ at position C. The rotation of LC directors decreases as the positions move toward the centers of electrodes and slits, where the maximum twist angle is $\sim 50^{\circ}$ at the intermediate positions $\mathrm{B}$ and $\mathrm{D}$, and is $\sim 30^{\circ}$ at central positions $\mathrm{A}$ and $\mathrm{E}$. Intuitively, the reduced transmittance originates from the inadequate rotation of $\mathrm{LC}$ directors near these centers.

To have a deeper understanding, the polarization changes of the normally incident light passing through this FFS cell from different positions (from A to E) are traced on the Poincaré sphere. ${ }^{13}$ Figure 3 plots their traces projected on the $S_{1}-S_{2}$ plane. Here, the bottom linear polarizer transmission axis $T_{\mathrm{btm}}$ is at a position with Stokes parameter $S_{1}$ $=+1$, and the transmission axis $T_{\text {top }}$ of the top linear polarizer is at $S_{1}=-1$. The final $S_{1}$ value indicates the transmittance of the light, i.e., if $S_{1}$ is closer to -1 , the transmittance would be larger. From Fig. 3, the light traversing the LC cell at position $\mathrm{C}$ has its final Stokes parameter $S_{1}$ closest to -1 , followed by $\mathrm{B}$ and $\mathrm{D}$, and then $\mathrm{A}$ and $\mathrm{E}$. These traces directly relate to the different LC director distributions. Referring to Fig. 2, at the bright state in a FFS cell, the LC profile looks like two serial TN LC cells with a reversed twist sense. The

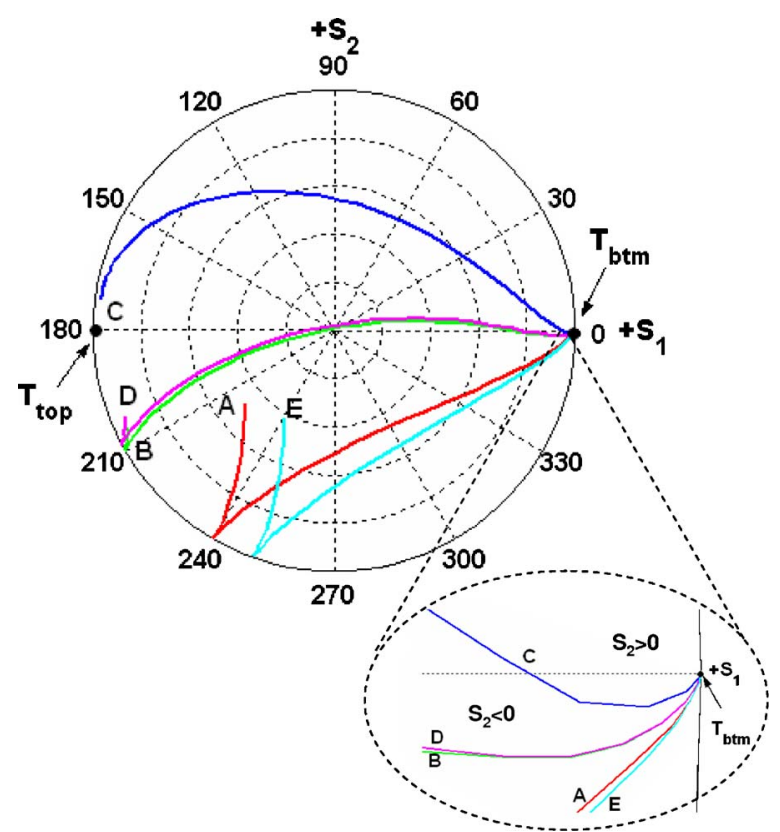

FIG. 3. (Color online) Polarization change traces projected on the $S_{1}-S_{2}$ plane for different incident positions. 


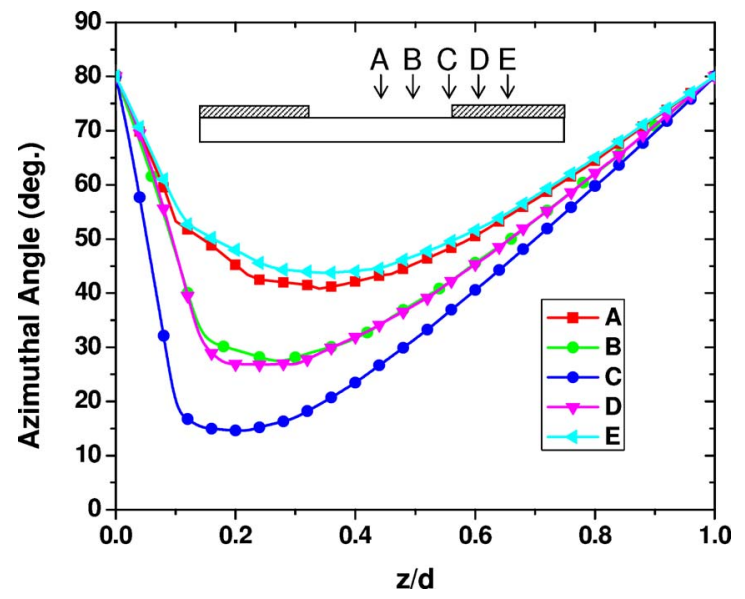

FIG. 4. (Color online) Azimuthal angle distributions at different cell positions with a negative chiral dopant having a chiral pitch $p=8 \mu \mathrm{m}$.

first clockwise twist profile very quickly occurs in a relatively shallow region, e.g., at position $\mathrm{C}$, the azimuthal angle changes from $80^{\circ}$ to $\sim 15^{\circ}$ within the $z \sim 0.2 d$ zone. For the light incident on position $\mathrm{C}$, the effective optic axis of the first $\sim 10 \%$ LC cell near the bottom surface $(z / d<0.1)$ is less than $-45^{\circ}$ with respect to the incident light (on Poincaré sphere, the rotational axis position should be $2 \times$ of the real angle, i.e., the optic axis here is confined between $270^{\circ}$ and $360^{\circ}$ in Fig. 3), rotating the light with its $S_{2}$ less than 0 , as shown in the expanded plot in Fig. 3. However, the following quick $65^{\circ}$ change of azimuthal angle makes the portion of LC layer having its effective optic axis more than $-45^{\circ}$ (the effective optic axis now is between $180^{\circ}$ and $270^{\circ}$ in Fig. 3) between the regions with $z / d \in(0.10,0.55)$. Thus, this portion of LC layer starts to rotate the light on the sphere surface with its $S_{2}$ greater than 0 . After this, the LC twist angle will gradually decrease back to a $0^{\circ}$ change at the top surface and the LC effective optic axis also returns to the $+S_{1}$ axis. However, this decrease of the twist angle effectively twists the final polarization back to the top polarizer transmission axis at $T_{\text {top }}$. Obviously, for light incident at positions A and E, the inadequate twist of LC directors near the bottom surface turns the LC layer's effective axis always less than $45^{\circ}$ to the incident light. The lights are rotated in a sense with their Stokes parameters $S_{2}$ always less than 0 , resulting in a far deviation of their final polarizations from the top polarizer's transmission axis.

From above analysis, we found the maximum LC twist angle plays a very important role affecting the transmittance of a FFS cell. In the conventional cell without any chiral dopant, the LC molecules at other positions such as B, C, and $\mathrm{D}$ can be reversely twisted by horizontal fields, but those near positions $\mathrm{A}$ and $\mathrm{E}$ with a weak horizontal field are mainly perturbed by the movement of neighboring molecules, as indicated in Fig. 1. Here, we propose to improve this clockwise LC rotation by adding a reversed-handed (or negative) chiral dopant. Figure 4 shows the LC director distribution at the same cell positions (from A to E) but with a negative chiral dopant whose pitch length is $p=8 \mu \mathrm{m}$. With such a chiral dopant, the azimuthal angle profiles at positions B, C, and D remains similar to those in Fig. 2, as they are mainly reoriented by the rich horizontal electric fields there. However, near the central regions (positions A and E) where the horizontal field is weak, the improvement is evident. These LC molecules are now easier to be perturbed by the adjacent LC directors, when the reverse helical twisting power from the chiral dopant has the same twist sense as the bottom surface LC molecules. Ultimately, their final polarization state is closer to the top linear polarizer's transmission axis resulting in a higher transmittance. The operation voltage of this $2 \mu \mathrm{m}$ FFS cell with a $2 \mu \mathrm{m}$ electrode width and $3 \mu \mathrm{m}$ electrode gap is about $5.0 \mathrm{~V}_{\mathrm{rms}}$, but its maximum transmittance is enhanced to $\sim 90.6 \%$ (as compared to $86.2 \%$ of a conventional cell without chiral dopant). The rise time of the $2 \mu \mathrm{m}$ cell is calculated to be $\sim 6.8 \mathrm{~ms}$ and decay time is $\sim 8.5 \mathrm{~ms}$. The negative chiral dopant helps LCs near the surface to better rotate, but it also increases the competition with the counterclockwise twist tendency induced by the top surface anchoring force. As a result, the overall driving voltage is slightly increased. In addition, this small amount of chiral dopant does not change the initial homogeneous LC profile, i.e., the surface anchoring force still dominates in such a thin LC cell. In experiment, we prepared a $2 \mu \mathrm{m}$ thin homogeneous cell filled with BL-036 LC mixture and a chiral dopant MLC-6248 whose pitch length was $8 \mu \mathrm{m}$. Under crossed linear polarizers, an excellent dark state was observed. Although reducing the pitch length to $4 \mu \mathrm{m}$ would further improve the transmittance $(\sim 94 \%)$, the helical twisting power might also be increased to overcome the LC surface anchoring energy so that the initial state of the LC cell at $V=0$ is altered. Further investigation on the relation between chiral dopant and the surface anchoring is undergoing.

In conclusion, we have demonstrated a thin FFS cell doped with a reverse-handed chiral compound with $d / p$ $\sim 0.25$. This cell exhibits a fast response time $(6-8 \mathrm{~ms}$ without overdrive), high transmittance, low operating voltage, and intrinsically wide viewing angle. This FFS cell is particularly attractive for LCD TV applications where image blurring is an important issue. The challenges are to develop high birefringence $(\Delta n \sim 0.2)$ LC materials and to perfect thin cell manufacturing technology. To archive high birefringence, fluorinated terphenyl LCs can be considered. ${ }^{14}$ To make thin cell, one drop fill technique needs to be stretched.

The authors are indebted to the financial support from Chi-Mei Optoelectronics, Taiwan, and S. Gauza and Y. Zhou for technical assistance.

${ }^{1}$ D. K. Yang and S. T. Wu, Fundamentals of Liquid Crystal Devices (Wiley, New York, 2006).

${ }^{2}$ S. T. Wu and U. Efron, Appl. Phys. Lett. 48, 624 (1986).

${ }^{3}$ S. Gauza, X. Zhu, W. Piecek, R. Dabrowski, and S. T. Wu, J. Disp. Technol. 3, 250 (2007).

${ }^{4}$ M. Jiao, Z. Ge, Q. Song, and S. T. Wu, Appl. Phys. Lett. 92, 061102 (2008).

${ }^{5}$ A. Takeda, S. Kataoka, T. Sasaki, H. Chida, H. Tsuda, K. Ohmuro, T. Sasabayashi, Y. Koike, and K. Okamoto, SID Int. Symp. Digest Tech. Papers 29, 1077 (1998).

${ }^{6}$ M. Ohe and K. Kondo, Appl. Phys. Lett. 67, 3895 (1995).

${ }^{7}$ H. Hong, H. Shin, and I. Chung, J. Disp. Technol. 3, 361 (2007).

${ }^{8}$ S. H. Lee, S. L. Lee, and H. Y. Kim, Appl. Phys. Lett. 73, 2881 (1998).

${ }^{9}$ M. Schadt and W. Helfrich, Appl. Phys. Lett. 18, 127 (1971).

${ }^{10}$ Y. M. Jeon, I. S. Song, S. H. Lee, H. Y. Kim, S. Y. Kim, and Y. J. Lim, SID Int. Symp. Digest Tech. Papers 36, 328 (1998).

${ }^{11}$ A. Lien, Appl. Phys. Lett. 57, 2767 (1990).

${ }^{12}$ Z. Ge, X. Zhu, T. X. Wu, and S. T. Wu, J. Opt. Soc. Am. A 22, 966 (2005).

${ }^{13}$ S. Huard, Polarization of Light (Wiley, New York, 1997).

${ }^{14}$ A. Parish, S. Gauza, S. T. Wu, J. Dziaduszek, and R. Dabrowski, Liq. Cryst. 35, 79 (2008). 